\title{
Representations of the Generalized Lie Algebra $\mathrm{sl}(2)_{\mathrm{q}}$
}

\author{
V.K. Dobrev ${ }^{1}$ and A. Sudbery ${ }^{2}$ \\ ${ }^{1}$ Bulgarian Academy of Sciences \\ Institute of Nuclear Research and Nuclear Energy \\ 72 Tsarigradsko Chaussee, 1784 Sofia, Bulgaria \\ dobrev@bgearn.acad.bg \\ ${ }^{2}$ University of York \\ Department of Mathematics \\ Heslington, York, England YO1 5DD \\ as2@york.ac.uk
}

\begin{abstract}
We construct finite-dimensional irreducible representations of two quantum algebras related to the generalized Lie algebra $\mathfrak{s l}(2)_{q}$ introduced by Lyubashenko and the second named author. We consider separately the cases of $q$ generic and $q$ at roots of unity. Some of the representations have no classical analog even for generic $q$. Some of the representations have no analog to the finite-dimensional representations of the quantised enveloping algebra $U_{q}(s l(2))$, while in those that do there are different matrix elements.
\end{abstract}

\section{Introduction}

A number of authors [1], [2], [3], [4] have suggested definitions of "quantum Lie algebras", 
the aim being to obtain structures which bear the same relation to quantised enveloping algebras as Lie algebras do to their enveloping algebras. It is of interest to determine the representations of such quantum Lie algebras, in those cases where a notion of "representation" is defined, and compare them to the classical representation theory. For generic values of the deformation parameter $q$ it is to be expected that the representations will resemble those of the classical Lie algebras which are deformed into the quantum versions, since the representation theory of a quantised enveloping algebra is essentially the same as that of the classical Lie algebra, but the details of this resemblance will help to illuminate the nature of a quantum Lie algebra. This relationship breaks down if $q$ is a root of unity, which is of much interest in physics, and it is therefore particularly significant to determine the representations of a quantum Lie algebra in this case.

In this paper we start on such a study by constructing finite-dimensional representations of the simplest example of the generalized Lie algebras introduced in [4. A representation of this algebra, in the sense defined in [4], is nothing but a representation of an associative algebra, the enveloping algebra of the quantum Lie algebra. This is obtained from a larger algebra with a central element by imposing a relation giving the central element as a function of Casimir-like elements. We investigate the representations also of this larger algebra, which is possibly more natural in the context of generalized Lie algebras, and find that it has additional one-dimensional representations.

The paper is organised as follows. In Section 2 we introduce explicitly the two quantum algebras which we consider. In Sections 3 and 4 we construct finite-dimensional representations of these algebras for generic values of $q$. In Sections 5 and 6 we consider the case when $q$ is at roots of unity. Section 7 contains a Summary of our results.

\section{The quantum Lie algebra $\mathfrak{s l}(2)_{q}$}

The generalized Lie algebra $\mathfrak{s l}(2)_{q}$ was introduced in [4], cf. also [5], [6], [7]. Its enveloping algebra $\mathcal{A} \equiv U\left(\mathfrak{s l}(2)_{q}\right)$ is defined by Eq. (3.5) of $\llbracket$. For the purposes of developing the representation theory it is enough to work with the algebras $\mathcal{B}, \mathcal{F}$, cf. [4]. The algebra 
$\mathcal{B}$ is generated by four generators: $X_{0}, X_{ \pm}, C$ with relations:

$$
\begin{gathered}
q^{2} X_{0} X_{+}-X_{+} X_{0}=q C X_{+} \\
q^{-2} X_{0} X_{-}-X_{-} X_{0}=-q^{-1} C X_{-} \\
X_{+} X_{-}-X_{-} X_{+}=\left(q+q^{-1}\right)\left(C-\lambda X_{0}\right) X_{0} \\
C X_{m}=X_{m} C, \quad m=0, \pm 1 \\
\quad \lambda \equiv q-q^{-1}
\end{gathered}
$$

The algebra $\mathcal{B}$ is related to the locally finite part $\mathcal{F}$ of the simply-connected quantised enveloping algebra $\bar{U}_{q}(s l(2))$. The algebra $\mathcal{F}$ was obtained in [4] from $\mathcal{B}$ by putting $C$ equal to a function of the second order Casimir:

$$
C_{2}=\left(q+q^{-1}\right) X_{0}^{2}+q X_{-} X_{+}+q^{-1} X_{+} X_{-}
$$

namely,

$$
C^{2}=1+\frac{\lambda^{2}}{q+q^{-1}} C_{2}
$$

For shortness we shall call $\mathcal{F}$ the restricted algebra. The enveloping algebra $\mathcal{A}$, on the other hand, is obtained by putting $C=1$ 四].

We shall need a triangular decomposition of $\mathcal{B}$ :

$$
\mathcal{B}=\mathcal{B}_{+} \otimes \mathcal{B}_{0} \otimes \mathcal{B}_{-}
$$

where $\mathcal{B}_{ \pm}$is generated by $X_{ \pm}$, while $\mathcal{B}_{0}$ is generated by $X_{0}, C$. We shall call the abelian Lie algebra $\mathcal{H}$ generated by $X_{0}, C$ the Cartan subalgebra of $\mathcal{B}$. Note that $\mathcal{B}_{0}$ is the enveloping algebra of $\mathcal{H}$. The same decomposition is used for the algebra $\mathcal{F}$ with the relation (2.3) enforced.

Further we shall analyse the algebras $\mathcal{B}$ and $\mathcal{F}$ separately.

\section{Highest weight representations}

Highest weight modules of $\mathcal{B}$ are standardly determined by a highest weight vector $v_{0}$ which is annihilated by the raising generator $X_{+}$and on which the Cartan generators act 
by the corresponding value of the highest weight $\Lambda \in \mathcal{H}^{*}$ :

$$
\begin{array}{rlrl}
X_{+} v_{0}= & 0 \\
H v_{0}= & \Lambda(H) v_{0}, & H \in \mathcal{H} \\
& M \equiv \Lambda\left(X_{0}\right), & c \equiv \Lambda(C)
\end{array}
$$

In particular, we shall be interested in Verma modules over $\mathcal{F}$. As in the classical case a Verma module $V^{\Lambda}$ is a highest weight module (HWM) of weight $\Lambda$ induced from one-dimensional representation of a Borel subalgebra $\tilde{\mathcal{B}}$, e.g., $\tilde{\mathcal{B}}=\mathcal{B}_{+} \otimes \mathcal{B}_{0}$, on the highest weight vector, e.g., $v_{0}$. As vector spaces we have:

$$
V^{\Lambda} \cong \mathcal{B} \otimes_{\tilde{\mathcal{B}}} v_{0}=\mathcal{B}_{-} \otimes v_{0}=\text { l.s. }\left\{v_{k} \equiv X_{-}^{k} \otimes v_{0} \mid k \in \mathbb{Z}_{+}\right\}
$$

where we have identified $1_{\mathcal{B}} \otimes v_{0}$ with $v_{0}$.

The action of the generators of $\mathcal{B}$ on the basis of $V^{\Lambda}$ is given as follows:

$$
\begin{aligned}
X_{+} v_{k}= & q^{2 k-2}(c-\lambda M)\left([2 k]_{q} M-q[k]_{q}[k-1]_{q} c\right) v_{k-1} \\
X_{-} v_{k}= & v_{k+1} \\
X_{0} v_{k}= & \left(q^{2 k} M-q^{k}[k]_{q} c\right) v_{k} \\
C v_{k}= & c v_{k} \\
& {[k]_{q} \equiv\left(q^{k}-q^{-k}\right) / \lambda }
\end{aligned}
$$

To obtain (3.3a,d) we have used the following calculations which follow from (2.1) :

$$
\begin{aligned}
X_{0} X_{-}^{k} & =X_{-}^{k}\left(q^{2 k} X_{0}-q^{k}[k]_{q} C\right) \\
{\left[X_{+}, X_{-}^{k}\right] } & =X_{-}^{k-1} q^{2 k-2}\left(C-\lambda X_{0}\right)\left([2 k]_{q} X_{0}-q[k]_{q}[k-1]_{q} C\right)
\end{aligned}
$$

As in the classical case the analysis of reducibility of Verma modules is an important tool in the representation theory. This analysis starts (cf. [8]) with the search for singular vectors. A singular vector $v_{s}$ of a Verma module $V^{\Lambda}$ is defined as follows: $v_{s} \in V^{\Lambda}$, $v_{s} \notin \mathscr{C} v_{0}$ and it satisfies the following properties (cf., e.g., [8]) :

$$
\begin{aligned}
X_{+} v_{s} & =0 \\
H v_{s} & =\Lambda^{\prime}(H) v_{s}, \quad H \in \mathcal{H}, \quad \Lambda^{\prime} \in \mathcal{H}^{*}
\end{aligned}
$$


First we note that since $C$ is central its value is the same as on $v_{0}: c^{\prime} \equiv \Lambda^{\prime}(C)=c$. Further, we proceed to find the possible singular vectors using that they are eigenvectors of $X_{0}$. But the eigenvectors of $X_{0}$ are $X_{-}^{n} \otimes v_{0}$, all with different eigenvalues. Thus, a singular vector will be given by the classical expression (omitting the overall normalization) : $v_{s}=X_{-}^{n} \otimes$ $v_{0}$ for some fixed $n \in \mathbb{N}$, and we have:

$$
X_{0} v_{s}=M^{\prime} v_{s}, \quad M^{\prime} \equiv \Lambda^{\prime}\left(X_{0}\right)=q^{2 n} M-q^{n}[n]_{q} c
$$

Finally, we have to impose (3.5a) for which we calculate (using (3.4b)) :

$$
X_{+} v_{s}=X_{-}^{n-1} q^{2 n-2}(c-\lambda M)\left([2 n]_{q} M-q[n]_{q}[n-1]_{q} c\right) \otimes v_{0}
$$

For the further analysis we suppose that the deformation parameter $q$ is not a nontrivial root of unity. Then there are two possibilities for (3.7) to be zero, and thus, we have two possibilities to fulfil (3.5a) :

$$
\begin{aligned}
M & =q[n]_{q}[n-1]_{q} c /[2 n]_{q} \\
c & =\lambda M
\end{aligned}
$$

We shall analyse the two possibilities in (3.8) separately since they have very different implications; moreover, they are incompatible unless $c=M=0$ when they coincide and which we shall treat as partial case of $(3.8 b)$.

3.1. The first possibility $(\underline{3.8 a})$ (with $c \neq 0$ ) corresponds to the classical relation between $n$ and the highest weight $\Lambda$ (obtained for $q, c \rightarrow 1): M=(n-1) / 2$. Thus, if we fix $n \in \mathbb{I}$ then $v_{s}=X_{-}^{n} \otimes v_{0}$ is a singular vector when $M$ has the value (3.8a). The shifted weight $\Lambda^{\prime}$ corresponds to another Verma module $V^{\Lambda^{\prime}}$ which is the naximal invariant submodule of $V^{\Lambda}$. The corresponding eigenvalue of $X_{0}$ is (cf. (3.6)):

$$
M^{\prime}=-q[n]_{q}[n+1]_{q} c /[2 n]_{q}
$$

Note that the Verma module $V^{\Lambda^{\prime}}$ does not have a singular vector. Indeed, there is no $n^{\prime} \in \mathbb{N}$ such that (3.8a) holds for the pair $\left(M^{\prime}, n^{\prime}\right)$ replacing $(M, n)$. Also (3.8b) can not hold for $M^{\prime}$ since $c=\lambda M^{\prime}$ will contradict (3.9). 
The factor-module $L_{n, c} \cong V^{\Lambda} / V^{\Lambda^{\prime}}$ is irreducible and finite-dimensional of dimension $n$. It has a highest weight vector $|n, c\rangle$ such that:

$$
\begin{aligned}
X_{+}|n, c\rangle & =0 \\
H|n, c\rangle & =\Lambda(H)|n, c\rangle, \quad H \in \mathcal{H} \\
X_{-}^{n}|n, c\rangle & =0
\end{aligned}
$$

Let us denote by $w_{k} \equiv X_{-}^{k}|n, c\rangle, k=0,1, \ldots, n-1$, the states of $L_{n, c}$. The transformation rules for $w_{k}$ are:

$$
\begin{aligned}
X_{+} w_{k} & =q^{2 k-n}[k]_{q}[n-k]_{q}\left(\frac{c[2]_{q}[n]_{q}}{[2 n]_{q}}\right)^{2} w_{k-1} \\
X_{-} w_{k} & =w_{k+1}, \quad k<n-1 \\
X_{-} w_{n-1} & =0 \\
X_{0} w_{k} & =\frac{c q^{k}[n]_{q}}{[2 n]_{q}}\left([n-k]_{q}-q^{1-n}[k+1]_{q}\right) w_{k} \\
C w_{k} & =c w_{k}
\end{aligned}
$$

Thus, the vector $w_{n-1}$ is the lowest weight vector of $L_{n, c}$.

Next we introduce a bilinear form in $L_{n, c}$ by the formula:

$$
\left(w_{j}, w_{k}\right) \equiv\left\langle n, c\left|X_{+}^{j} X_{-}^{k}\right| n, c\right\rangle
$$

where $\langle n, c|$ is such that $\langle n, c|| n, c\rangle=1$ and:

$$
\begin{aligned}
\langle n, c| X_{-} & =0 \\
\langle n, c| H & =\Lambda(H)\langle n, c|, \quad H \in \mathcal{H} \\
\langle n, c| X_{+}^{n} & =0
\end{aligned}
$$

Then we obtain :

$$
\begin{gathered}
\left(w_{j}, w_{k}\right)=\delta_{j k} q^{k(k+1-n)} \frac{[k]_{q} ![n-1]_{q} !}{[n-1-k]_{q} !}\left(\frac{c[2]_{q}[n]_{q}}{[2 n]_{q}}\right)^{2 k} \\
{[k]_{q} ! \equiv[k]_{q}[k-1]_{q} \ldots[1]_{q}, \quad[0]_{q} ! \equiv 1}
\end{gathered}
$$

Clearly, (3.14) is real-valued for real $q, c$. Thus, for $q, c \in \mathbb{R}$ we can turn (3.12) into a scalar product and define the norm of the basis vectors:

$$
\left|w_{k}\right| \equiv \sqrt{\left(w_{k}, w_{k}\right)}=q^{k(k+1-n) / 2} \sqrt{\frac{[k]_{q} ![n-1]_{q} !}{[n-1-k]_{q} !}}\left(\frac{c[2]_{q}[n]_{q}}{[2 n]_{q}}\right)^{k}
$$


where have chosen the root that is positive for positive $c, q$. We can also introduce orthonormal basis:

$$
u_{k} \equiv \frac{1}{\left|w_{k}\right|} w_{k}
$$

Then we have:

$$
\left(u_{j}, u_{k}\right)=\delta_{j k}
$$

The transformation rules for the basis vectors $u_{k}$ are:

$$
\begin{aligned}
X_{+} u_{k} & =q^{k-n / 2} \sqrt{[k]_{q}[n-k]_{q}} \frac{c[2]_{q}[n]_{q}}{[2 n]_{q}} u_{k-1} \\
X_{-} u_{k} & =q^{k+1-n / 2} \sqrt{[n-1-k]_{q}[k+1]_{q}} \frac{c[2]_{q}[n]_{q}}{[2 n]_{q}} u_{k+1} \\
X_{0} u_{k} & =\frac{c q^{k}[n]_{q}}{[2 n]_{q}}\left([n-k]_{q}-q^{1-n}[k+1]_{q}\right) u_{k} \\
C u_{k} & =c u_{k}
\end{aligned}
$$

The above scalar product is invariant under the real form $\mathcal{B}_{r}$ of $\mathcal{B}$ defined by the antilinear antiinvolution:

$$
\omega\left(X^{ \pm}\right)=X^{\mp}, \quad \omega\left(X_{0}\right)=X_{0}, \quad \omega(C)=C
$$

Indeed, the algebraic relations (2.1) are preserved by $\omega$ for real $q$. The $\mathcal{B}_{r}$ invariance of the scalar product means that:

$$
\left(w_{j}, X w_{k}\right)=\left(\omega(X) w_{j}, w_{k}\right), \quad X \in \mathcal{B}
$$

which is automatically satisfied with the definition (3.12). (Note that (3.20) defines $($, ) as the Shapovalov bilinear form [9].)

Thus, for every $n \in \mathbb{N}$ we have constructed $n$-dimensional irreducible representations (irreps) of $\mathcal{B}$ parametrized by $c \in \mathbb{C}, c \neq 0$, with basis $w_{k}$ or $u_{k},(k=0, \ldots, n-1)$. For $q, c \in \mathbb{R}$ these are irreps of the real form $\mathcal{B}_{r}$, which are unitary when $q, c>0$.

3.2. The second possibility (3.8b) has no classical analogue. It tells us that if $c$ and $M$ are related as in (3.8b) then each vector of the basis of $V^{\Lambda}$ is a singular vector. Moreover, 
all of them have the same weight since $M^{\prime}=M$, cf. (3.6). This is clear also from the transformation rules (3.3) when $c=\lambda M$ :

$$
\begin{aligned}
X_{+} v_{k} & =0 \\
X_{-} v_{k} & =v_{k+1} \\
X_{0} v_{k} & =M v_{k} \\
C v_{k} & =\lambda M v_{k}
\end{aligned}
$$

Clearly, we have an infinite sequence of embedded reducible Verma modules $V_{n}=$ l.s. $\left\{v_{k} \mid k \in \mathbb{Z}_{+}, \quad k \geq n\right\}$ for $n \in \mathbb{Z}_{+}$as follows: $V_{n} \supset V_{n+1}$, the latter being the maximal invariant submodule of the former. Note that $V_{n}$ is isomorphic to a submodule of all $V_{m}$ with $n>m$. Furthermore, because of the coincidence of the weights these modules are also all isomorphic to each other: $V_{n} \cong V_{m}$ for all $m, n$. It is also clear that for every $M$ there is only one irreducible module, namely the one-dimensional $L_{M} \cong V_{n} / V_{n+1}$, for any $n$. Denoting by $|M\rangle$ the only state in $L_{M}$ we have for the action on it:

$$
\begin{aligned}
X_{+}|M\rangle & =0 \\
X_{-}|M\rangle & =0 \\
X_{0}|M\rangle & =M|M\rangle \\
C|M\rangle & =\lambda M|M\rangle
\end{aligned}
$$

Note that the above one-dimensional irrep is different from the one-dimenional $L_{1, c}$ from the previous subsection. Indeed, though the action of $X_{ \pm}$is the same, the ratio of eigenvalues of $C$ to $X_{0}$ here is $\lambda$, while there it is $-[2]_{q} / q$.

\section{Highest weight representations of the restricted algebra}

The highest weight representations of the restricted algebra $\mathcal{F}$ are obtained from those of $\mathcal{B}$ imposing the relation (2.3). In particular, there is the following relation between the values of the Cartan generators:

$$
c^{2}=1+\lambda^{2}\left(\frac{M^{2}}{q^{2}}+c \frac{M}{q}\right)
$$


This relation has to be imposed on all formulae of the previous Section. There are no essential consequences of this for the generic Verma modules. For the reducible Verma modules there are more interesting consequences. First we notice that the reducibility condition (3.8b) is incompatible with (4.1), and thus there would be no special one-dimensional irreps like $L_{M}$, cf. (3.22.) So it remains to consider the combination of the reducibility condition (3.8a) with (4.1) from which we obtain that:

$$
c=\frac{\epsilon[2 n]_{q}}{[2]_{q}[n]_{q}}, \quad M=\frac{q[n]_{q}[n-1]_{q} c}{[2 n]_{q}}=\frac{\epsilon q[n-1]_{q}}{[2]_{q}}, \quad \epsilon= \pm 1
$$

In this case the analogue of (3.9) is:

$$
M^{\prime}=-\epsilon q[n+1]_{q} /[2]_{q}
$$

Let us denote the finite-dimensional representations of $\mathcal{F}$ by $\tilde{L}_{n, \epsilon}$ and the basis by $\tilde{w}_{k}$, $k=0, \ldots, n-1$. The transformation rules are:

$$
\begin{aligned}
X_{+} \tilde{w}_{k} & =q^{2 k-n}[k]_{q}[n-k]_{q} \tilde{w}_{k-1} \\
X_{-} \tilde{w}_{k} & =\tilde{w}_{k+1}, \quad k<n-1 \\
X_{-} \tilde{w}_{n-1} & =0 \\
X_{0} \tilde{w}_{k} & =\frac{\epsilon q^{k}}{[2]_{q}}\left([n-k]_{q}-q^{1-n}[k+1]_{q}\right) \tilde{w}_{k} \\
C \tilde{w}_{k} & =\frac{\epsilon[2 n]_{q}}{[2]_{q}[n]_{q}} \tilde{w}_{k}
\end{aligned}
$$

Further, the analogues of (3.14) and (3.15) are:

$$
\begin{gathered}
\left(\tilde{w}_{j}, \tilde{w}_{k}\right)=\delta_{j k} q^{k(k+1-n)} \frac{[k]_{q} ![n-1]_{q} !}{[n-1-k]_{q} !} \\
\left|\tilde{w}_{k}\right| \equiv \sqrt{\left(\tilde{w}_{k}, \tilde{w}_{k}\right)}=q^{k(k+1-n) / 2}[k]_{q} ! \sqrt{\frac{[k]_{q} ![n-1]_{q} !}{[n-1-k]_{q} !}}
\end{gathered}
$$

We can also introduce orthonormal basis:

$$
\tilde{u}_{k} \equiv \frac{1}{\left|\tilde{w}_{k}\right|} \tilde{w}_{k}, \quad\left(\tilde{u}_{j}, \tilde{u}_{k}\right)=\delta_{j k}
$$


for which the transformation rules are:

$$
\begin{aligned}
X_{+} \tilde{u}_{k} & =q^{k-n / 2} \sqrt{[k]_{q}[n-k]_{q}} \tilde{u}_{k-1} \\
X_{-} \tilde{u}_{k} & =q^{k+1-n / 2} \sqrt{[n-1-k]_{q}[k+1]_{q}} \tilde{u}_{k+1} \\
X_{0} \tilde{u}_{k} & =\frac{\epsilon q^{k}}{[2]_{q}}\left([n-k]_{q}-q^{1-n}[k+1]_{q}\right) \tilde{u}_{k} \\
C \tilde{u}_{k} & =\frac{\epsilon[2 n]_{q}}{[2]_{q}[n]_{q}} \tilde{u}_{k}
\end{aligned}
$$

Thus, for every $n \in \mathbb{N}$ we have constructed $n$-dimensional irreducible representations of $\mathcal{F}$ parametrized by $\epsilon= \pm 1$, with bases $\tilde{w}_{k}$ or $\tilde{u}_{k},(k=0, \ldots, n-1)$.

\section{Highest weight representations at roots of unity}

Here we consider representations of the algebra $\mathcal{B}$ in the case when the deformation parameter is at roots of unity. More precisely, first we consider the cases when $q^{2}$ is a primitive $N$-th root of unity: $q=e^{\pi i / N}, N \in \mathbb{N}+1$. Then we have:

$$
[x]_{q}=\frac{\sin \pi x / N}{\sin \pi / N}
$$

In such cases there are additional reducibility conditions coming from (3.7) besides (3.8a,b). For this we rewrite $(3.8 a)$ in a more general fashion:

$$
M[2 n]_{q}=q[n]_{q}[n-1]_{q} c
$$

Then we note that from (5.1) follows that $[N]_{q}=[2 N]_{q}=0$, so $\left(\overline{3.8 a^{\prime}}\right)$ is satisfied for $n \rightarrow N$. Thus, $v_{s}^{N}=X_{-}^{N} \otimes v_{0}$ is a singular vector independently of the highest weight $\Lambda$. Similarly to the analysis done in [10] for the quantised enveloping algebral $U_{q}(\operatorname{sl}(2))$ all $v_{s}^{p N}=X_{-}^{p N} \otimes v_{0}$ for $p \in \mathbb{N}$ are singular vectors. The Verma modules they realize we denote by $\tilde{V}_{p}, p \in \mathbb{Z}_{+}, \tilde{V}_{0} \equiv V^{\Lambda}$. These are embedded reducible Verma modules $\tilde{V}_{p} \supset \tilde{V}_{p+1}$ with the same highest weight $\Lambda$. Indeed, for any $\tilde{V}_{p}$ using (3.6) with $n \rightarrow p N$ we have: $M^{\prime}=q^{2 p N} M-q^{p N}[p N]_{q} c=M$.

1 We recall that though the quantised enveloping algebras $U_{q}(\mathcal{G})$ ) were introduced for arbitrary simple Lie algebras $\mathcal{G}$ in [11], [12], the example of $U_{q}(s l(2))$ was introduced in [13] as an algebra and in [14] as a Hopf algebra. 
The further analysis depends on whether there are additional singular vectors besides those just displayed. There are four cases.

5.1. We start with the case when $M, c$ do not satisfy either of (3.8a,b). We also suppose that $c \neq 0$ when $N$ is even. Then there are no additional singular vectors and there is only one irreducible $N$-dimensional $\operatorname{HWM} L_{\Lambda, N} \cong \tilde{V}_{p} / \tilde{V}_{p+1}$ (for any $p$ ), parametrized by all pairs $M, c$ not satisfying (3.8a,b). The action of the generators of $\mathcal{B}$ on the basis of $L_{\Lambda, N}$, which we denote by $\tilde{v}_{k},(k=0, \ldots, N-1)$, is given as follows:

$$
\begin{aligned}
X_{+} \tilde{v}_{k} & =q^{2 k-2}(c-\lambda M)\left([2 k]_{q} M-q[k]_{q}[k-1]_{q} c\right) \tilde{v}_{k-1} \\
X_{-} \tilde{v}_{k} & =\tilde{v}_{k+1}, \quad k<N-1 \\
X_{-} \tilde{v}_{N-1} & =0 \\
X_{0} \tilde{v}_{k} & =\left(q^{2 k} M-q^{k}[k]_{q} c\right) \tilde{v}_{k} \\
C \tilde{v}_{k} & =c \tilde{v}_{k}
\end{aligned}
$$

However, unlike the D-J case, these finite-dimensional representations are not unitarizable, which is easily seen if one considers the analogue of the bilinear form (3.12).

5.2. Next we consider the case when $M, c$ satisfy (3.8a) for some $n \in \mathbb{N}, n<N$. We also suppose that $c \neq 0$ (for any $N$ ). First we note that $n<N$ is not a restriction, since then (3.8d) holds also for all $n+p N, p \in \mathbb{Z}$. Indeed, we have:

$$
\begin{aligned}
& q[n+p N]_{q}[n+p N-1]_{q} c /[2(n+p N)]_{q}=q[n]_{q}[n-1]_{q} \cos ^{2}(\pi p) c /[2 n]_{q} \cos (2 \pi p)= \\
& =q[n]_{q}[n-1]_{q} c /[2 n]_{q}=M
\end{aligned}
$$

Thus, we have another infinite series of singular vectors $v_{s}^{\prime p N}=X_{-}^{n+p N} \otimes v_{0}$ for $p \in \mathbb{Z}_{+}$. They realize reducible Verma modules which we denote by $\tilde{V}_{p}^{\prime}, p \in \mathbb{Z}_{+} ;\left(\tilde{V}_{0}^{\prime}\right.$ is the analogue of $V^{\Lambda^{\prime}}$ introduced in the non-root-of-unity case, but here it is reducible). They all have the same highest weight $\Lambda^{\prime}$ determined by $M^{\prime}, c$ with $M^{\prime}$ given by (3.6). Indeed, substituting $n$ with $n+p N$ does not change the value of $M^{\prime}$ :

$$
\begin{aligned}
& q^{2(n+p N)} M-q^{n+p N}[n+p N]_{q} c=q^{2 n} M-q^{n+p N} e^{\pi i p}[n]_{q} \cos (\pi p) c= \\
& =q^{2 n} M-q^{n}[n]_{q} c=M^{\prime}
\end{aligned}
$$

Of course, after substituting $M$ with its value from (3.8a) we obtain the expression for $M^{\prime}$ in (3.9). We have the following infinite embedding chain:

$$
V^{\Lambda} \equiv \tilde{V}_{0} \supset \tilde{V}_{0}^{\prime} \supset \tilde{V}_{1} \supset \tilde{V}_{1}^{\prime} \supset \ldots
$$


where all embeddings are non-composite: the embeddings $\tilde{V}_{p} \supset \tilde{V}_{p}^{\prime}$ are realized by singular vectors: $X_{-}^{n} \otimes v_{p}, v_{p}$ being the highest weight vector of $\tilde{V}_{p}$, while the embeddings $\tilde{V}_{p}^{\prime} \supset \tilde{V}_{p+1}$ are realized by singular vectors: $X_{-}^{N-n} \otimes v_{p}^{\prime}, \quad v_{p}^{\prime}$ being the highest weight vector of $\tilde{V}_{p}^{\prime}$.

Now, factorizing each reducible Verma module by its maximal invariant submodule we obtain that for each $n \in \mathbb{I}, n<N$ there are two finite dimensional irreps parametrized by $c \in \mathbb{C}, c \neq 0: \quad L_{n, N} \cong \tilde{V}_{p} / \tilde{V}_{p}^{\prime} \quad$ (for any $p$ ) which is $n$-dimensional, and $L_{n, N}^{\prime} \cong$ $\tilde{V}_{p}^{\prime} / \tilde{V}_{p+1}$ (for any $p$ ) which is $(N-n)$-dimensional. However, it turns out that the irreps from one series are isomorphic to those of the other: $L_{n, N}^{\prime} \cong L_{N-n, N}$. Indeed, note that the value of $M^{\prime}$ for the Verma modules $\tilde{V}_{p}^{\prime}$ given by (3.9) should be obtained (for consistency) also from the formula for $M$ with $n$ substituted by $N-n$ (since this is the reducibility condition w.r.t. the non-composite singular vector $X_{-}^{N-n} \otimes v_{p}^{\prime}$ ) and indeed this is the case:

$$
\begin{aligned}
& q[N-n]_{q}[N-n-1]_{q} c /[2(N-n)]_{q}=-q[n-N]_{q}[n+1-N]_{q} c /[2(n-N)]_{q}= \\
& =-q[n]_{q}[n+1]_{q} c \cos ^{2}(\pi N) /[2 n]_{q} \cos (2 \pi N)=-q[n]_{q}[n+1]_{q} c /[2 n]_{q}=M^{\prime}
\end{aligned}
$$

Furthermore, the transformation rules for $L_{n, N}$ are the same as for $L_{n, c}$, cf. (3.11), while the transformation rules for $L_{n, N}^{\prime}$ are obtained from (3.11) by substituting $n \rightarrow N-n$.

Thus, we are left with one series of finite-dimensional irreps $L_{n, N}$.

5.3. Next, we consider the case when $M, c$ satisfy (3.8b) for arbitrary $c$. Actually, nothing is changed from the non-root-of-unity case since the relevant formulae (3.21) and (3.22) are not changed.

5.4. Finally, we consider the case when $N$ is even and $c=0$. Let $\tilde{N}=N / 2 \in \mathbb{N}$. In these cases there are additional reducibility conditions coming from (3.8a $)$. Indeed, from (5.1) follows that $[2 \tilde{N}]_{\tilde{q}}=0$ and $[\tilde{N}]_{\tilde{q}} \neq 0$. But if $c=0$ then $(3.8 a f)$ is again satisfied. Thus, the vector $\hat{v}_{s}^{\tilde{N}}=X_{-}^{\tilde{N}} \otimes v_{0}$ is a singular vector independently of the value of $M$. Similarly to the analysis of the first subsection also all $\hat{v}_{s}^{p \tilde{N}}=X_{-}^{p \tilde{N}} \otimes v_{0}$ for $p \in \mathbb{N}$ are singular vectors. Note that for $p$ even these are the singular vectors that we already have: $\hat{v}_{s}^{p \tilde{N}}=v_{s}^{\tilde{p} N}, \tilde{p}=p / 2$. The Verma modules they realize we denote by $\hat{V}_{p}$, $p \in \mathbb{Z}_{+}, \hat{V}_{0} \equiv V^{\Lambda}$. These are embedded reducible Verma modules $\hat{V}_{p} \supset \hat{V}_{p+1}$ with the same value of $M$ up to sign. Indeed, for any $\hat{V}_{p}$ using (3.6) with $n \rightarrow p \tilde{N}$ we have: 
$M^{\prime}=q^{2 p \tilde{N}} M-q^{p \tilde{N}}[p \tilde{N}]_{q} c=(-1)^{p} M$. Certainly, for even $p$ these are Verma modules from the first subsection: $\hat{V}_{p}=V_{\tilde{p}}$.

As above the further analysis depends on whether $M, c$ satisfy some of $(3.8 a, b)$. However, since $c=0$ then the only additional possibility is that also $M=0$, which is a partial case of $(\overline{3.8 b})$, which was considered in the previous subsection. Thus, further, we suppose that $M, c$ do not satisfy either of $(3.8 a, b)$ and that $M \neq 0$.

Then there are no additional singular vectors besides $\hat{v}_{s}^{p \tilde{N}}$. Then for each $M \neq 0$ there is only one irreducible $\mathrm{HWM} L_{M, \tilde{N}} \cong \hat{V}_{p} / \hat{V}_{p+1}$ (for any $p$ ) which is $\tilde{N}$-dimensional. The action of the generators of $\mathcal{B}$ on the basis of $L_{M, \tilde{N}}$, which we denote by $\hat{v}_{k},(k=$ $0, \ldots, \tilde{N}-1)$, is given as follows:

$$
\begin{aligned}
X_{+} \hat{v}_{k} & =-q^{2 k-2} \lambda[2 k]_{q} M^{2} \hat{v}_{k-1} \\
X_{-} \hat{v}_{k} & =\hat{v}_{k+1}, \quad k<\tilde{N}-1 \\
X_{-} \hat{v}_{\tilde{N}-1} & =0 \\
X_{0} \hat{v}_{k} & =q^{2 k} M \hat{v}_{k} \\
C \hat{v}_{k} & =0
\end{aligned}
$$

Note that if $\tilde{N}$ is odd it seems that formulae (5.6) may be obtained from (5.2) for $N$ odd and $c=0$ by the substitution $N \rightarrow \tilde{N}$. However, this is not the same irrep since with the same replacement the parameter $q$ there becomes $e^{\pi i / N} \rightarrow e^{\pi i / \tilde{N}}$ while the parameter $q$ here is $e^{\pi i / 2 \tilde{N}}$.

\section{Highest weight representations at roots of unity of the restricted algebra}

Here we consider representations of the restricted algebra $\mathcal{F}$ in the case when the deformation parameter is at roots of unity. We start with the case: $q=e^{\pi i / N}, N \in \mathbb{N}+1$, and so (3.8a') holds. The analysis is as for the algebra $\mathcal{B}$ but imposing the relation (4.1), i.e., combining the considerations of the previous two Sections.

6.1. We start with the case when $M, c$ do not satisfy (3.8a), i.e., (4.2) does not hold. We also suppose that $c \neq 0$ when $N$ is even. Then there is only one irreducible $N$ dimensional HWM parametrized by $M, c$ related by (4.1), which irrep we denote by $\tilde{L}_{\Lambda, N}$. For the transformation rules we can use formulae (5.2) with (4.1) imposed. 
6.2. Next we consider the case when $M, c$ satisfy (3.8a) and $c \neq 0$. Here we should be nore careful so we replace $n$ by $n+p N$ with $n<N$. Combining the reducibility condition (3.8a) with (4.1) we first obtain that:

$$
c^{2}=\frac{[2(n+p N)]_{q}^{2}}{[2]_{q}^{2}[n+p N]_{q}^{2}}=\frac{[2 n]_{q}^{2}}{[2]_{q}^{2}[n]_{q}^{2}}
$$

Then we recover (4.2) and (4.3) for $n<N$ which means that we have the same situation as for the unrestricted algebra at roots of unity. Thus, for each $n \in \mathbb{I}, n<N$ and $\epsilon= \pm 1$ there is a finite dimensional irrep: $\tilde{L}_{n, \epsilon, N}$ which is $n$-dimensional. The transformation rules for $\tilde{L}_{n, \epsilon, N}$ are the same as in the non-root-of-unity case, cf. (4.4).

6.3. Finally, we consider the case when $N$ is even and $c=0$. Let $\tilde{N}=N / 2 \in \mathbb{N}$. As for the unrestricted algebra there are additional reducibility conditions, i.e., again the vector $v_{s}^{\tilde{N}}=X_{-}^{\tilde{N}} \otimes v_{0}$ is a singular vector. However, because of (4.1) the value of $M^{2}$ is fixed:

$$
M^{2}=-\tilde{q}^{2} / \lambda^{2}, \quad M=\epsilon i \tilde{q} / \lambda, \quad \epsilon= \pm 1
$$

Otherwise, the analysis goes through and there is only one irreducible $\tilde{N}$-dimensional HWM $\tilde{L}_{\epsilon, \tilde{N}}$ parametrized by $\epsilon$. The action of the generators of $\mathcal{B}$ on the basis of $\tilde{L}_{\epsilon, \tilde{N}}$, which we denote by $\hat{v}_{k}^{\prime},(k=0, \ldots, \tilde{N}-1)$, is given as follows:

$$
\begin{aligned}
X_{+} \hat{v}_{k}^{\prime} & =\frac{\tilde{q}^{2 k}[2 k]_{\tilde{q}}}{\lambda} \hat{v}_{k-1}^{\prime} \\
X_{-} \hat{v}_{k}^{\prime} & =\hat{v}_{k+1}^{\prime}, \quad k<\tilde{N}-1 \\
X_{-} \hat{v}_{\tilde{N}-1}^{\prime} & =0 \\
X_{0} \hat{v}_{k}^{\prime} & =\frac{\epsilon i \tilde{q}^{2 k+1}}{\lambda} \hat{v}_{k}^{\prime} \\
C \hat{v}_{k}^{\prime} & =0
\end{aligned}
$$

The crucial feature of these two irreps is that they do not have a classical limit for $\tilde{q} \rightarrow 1$ (obtained for $N \rightarrow \infty$ ).

\section{Summary}

Below by $q$ generic we shall understand that $q$ is a nonzero complex number which is not a nontrivial root of unity. We have constructed the following finite-dimensional irreps of the algebras $\mathcal{B}$ and $\mathcal{F}$. 
7.1. For the algebra $\mathcal{B}$ :

- $L_{n, c}, n \in \mathbb{N}, c \in \mathbb{C}, c \neq 0, q$ generic, $\operatorname{dim} L_{n, c}=n$, cf. (3.11), (3.18).

- $L_{M}, M \in \mathbb{C}, \quad c=\lambda M, q$ arbitrary, $\operatorname{dim} L_{M}=1$, cf. (3.22).

- $L_{\Lambda, N}, N \in \mathbb{N}+1, q=e^{\pi i / N}, M, c \in \mathbb{C}$ arbitrary not satisfying (3.8a,b),$c \neq 0$ for $N$ even, $\operatorname{dim} L_{\Lambda, N}=N$, cf. (5.2).

- $L_{n, c, N}, n, N \in \mathbb{N}, n<N, q=e^{\pi i / N}, c \in \mathbb{C}, c \neq 0, \operatorname{dim} L_{n, c, N}=n$, cf. (3.11).

- $L_{M, \tilde{N}}, \quad N=2 \tilde{N} \in 2 \mathbb{N}, q=e^{\pi i / N}, \quad M \in \mathbb{C}, M \neq 0, \quad c=0, \quad \operatorname{dim} L_{M, \tilde{N}}=\tilde{N}$, cf. (6.3).

7.2. For the algebra $\mathcal{B}_{r}$ with $q \in \mathbb{R}, q \neq 0$ :

- $L_{n, c}, n \in \mathbb{N}, c \in \mathbb{R}, c \neq 0, \operatorname{dim} L_{n, c}=n$, cf. (3.11), (3.18); unitary for $q, c>0$.

7.3. For the algebra $\mathcal{F}$ :

- $\tilde{L}_{n, \epsilon}, n \in \mathbb{N}, \epsilon= \pm 1, q$ generic, $\operatorname{dim} L_{n, c}=n$, cf. (4.4), (4.8).

- $\tilde{L}_{\Lambda, N}, N \in \mathbb{N}+1, q=e^{\pi i / N}, M, c \in \mathbb{C}$ related by (4.1) and not satisfying (3.8a, $)$, $c \neq 0$ for $N$ even, $\operatorname{dim} L_{\Lambda, N}=N$, cf. (5.2).

- $\tilde{L}_{n, \epsilon, N}, n, N \in \mathbb{N}, n<N, q=e^{\pi i / N}, \epsilon= \pm 1, \operatorname{dim} \tilde{L}_{n, \epsilon, N}=n$, cf. (4.4).

- $\tilde{L}_{\epsilon, \tilde{N}}, N=2 \tilde{N} \in 2 N, q=e^{\pi i / N}, \operatorname{dim} \tilde{L}_{\epsilon, \tilde{N}}=\tilde{N}$, cf. (6.3).

7.4. Of the above irreps only $L_{n, c}$ and $\tilde{L}_{n, \epsilon}$ have classical $s l(2), s u(2)$ counterparts. For fixed $n$ for both cases this is the $n$-dimensional HWM of $s l(2)$ or $s u(2)$ with the conjugation $\omega$. The latter HWM is obtained from $L_{n, c}, \tilde{L}_{n, \epsilon}$, resp., for $q, c \rightarrow 1, q, \epsilon \rightarrow 1$, resp.

7.5. Of the above irreps all but $L_{M}, L_{M, \tilde{N}}, \tilde{L}_{\epsilon, \tilde{N}}$ have analogs in the representation theory [10] of the quantised enveloping algebra $U_{q}(s l(2))$. However, the matrix elements there are given by expressions different from ours. 
Acknowledgments. This work was supported by a Royal Society Grant. 


\section{References}

[1] S. Majid, Quantum and braided Lie algebras, J. Geom. Phys. 13 (1994) 307-356.

[2] G.W. Delius and A. Hüffmann, On quantum Lie algebras and quantum root systems, J. Phys. A: Math. Gen. 29 (1996) 1703-1722.

[3] G.W. Delius, A. Hüffmann, M. D. Gould and Y.-Z. Zhang, Quantum Lie algebras associated to $U_{q}\left(g l_{n}\right)$ and $U_{q}\left(s l_{n}\right)$, J. Phys. A: Math. Gen. 29 (1996) 5611-5617.

[4] V. Lyubashenko and A. Sudbery, Quantum Lie Algebras of Type $A_{n}$, q-alg/9510004, J. Math. Phys., to appear.

[5] A. Sudbery, The quantum orthogonal mystery, in: "Quantum Groups: Formalism and Applications", eds. J. Lukierski et al (Polish Scientific Publishers PWN, 1995), pp. 303-316.

[6] A. Sudbery, $S U_{q}(n)$ gauge theory, Phys. Lett. 375B (1996) 75-80.

[7] A. Sudbery, Quantum-group gauge theory, in: "Quantum Group Symposium at Group21", Proceedings of a Symposium at the XXI Intern. Colloquium on Group Theoretical Methods in Physics, (Goslar, July 1996), eds. H.-D. Doebner et al (Heron Press, Sofia, 1997) pp. 45-52.

[8] I.N. Bernstein, I.M. Gel'fand and S.I. Gel'fand, Structure of representations generated by highest weight vectors. Funkts. Anal. Prilozh. 5 (1), 1-9 (1971); English translation: Funkt. Anal. Appl. 5, 1-8 (1971)

[9] N.N. Shapovalov, On a bilinear form on the universal enveloping algebra of a complex semisimple Lie algebra, Funkts. Anal. Prilozh. 6 (4) (1972) 65-70; English translation: Funkt. Anal. Appl. 6 (1972) 307-312.

[10] V.K. Dobrev, Multiplet classification of highest weight modules over quantum universal enveloping algebras : the $U_{q}(s l(3, \mathscr{C}))$ example, in: Proceedings of the International Group Theory Conference (St. Andrews, 1989), eds. C.M. Campbell et al, Vol. 1, London Math. Soc. Lecture Note Series 159 (Cambridge University Press, 1991) pp. 87-104.

[11] V.G. Drinfeld, Hopf algebras and the quantum Yang-Baxter equation, Dokl. Akad. Nauk SSSR 283 (1985) 1060-1064 (in Russian); English translation: Soviet. Math. Dokl. 32 (1985) 254-258; Quantum groups, in: Proceedings ICM 1986, (MSRI, Berkeley, 1987) pp. 798-820.

[12] M. Jimbo, A $q$-difference analogue of $U(\mathcal{G})$ and the Yang-Baxter equation, Lett. Math. Phys. 10 (1985) 63-69; A q-difference analogue of $U(g l(N+1))$, Hecke algebras and the Yang-Baxter equation, Lett. Math. Phys. 11 (1986) 247-252.

[13] P.P. Kulish and N.Yu. Reshetikhin, The quantum linear problem for the sine-Gordon equation and higher representations, Zap. Nauch. Semin. LOMI 101 (1981) 101-110 (in Russian); English translation: J. Soviet. Math. 23 (1983) 2435-2441.

[14] E.K. Sklyanin, On an algebra generated by quadratic relations, Uspekhi Mat. Nauk 40 (1985) 214 (in Russian). 